\title{
The use of cerclage view and locking plates in cats with tibial fracture-two case reports
}

\begin{abstract}
Fractures, in particular tibial fractures, form a large part of the surgical caseload in small animals. Various methods of fixation have been described, including the use of cerclage wire and plates. However, there are few studies of bone stabilization in cats using these two implants. When used in combination cerclage wires compress the fracture site while the neutralization plate provides support. This paper reports two cases of tibial osteosynthesis in cats using cerclage wire and neutralizing compression plates. Primary healing of fractures in the distal third of the tibia occurred with the use of cerclage wires in combination with a neutralization locking compression plate (LCP) allowing early return to function of the fractured leg. The medical and radiographic records of cats with long oblique tibial fractures treated with cerclage wire and a neutralization locking compression plate (LCP) were examined. In one cases bone healing with formation of primary bone callus occurred with the use of cerclage wires and a neutralization plate.
\end{abstract}

Keywords: cat, tibial fracture, locking compression plate, cerclage wire
Volume I Issue 4 - 2017

\author{
Danilo Roberto Custódio Marques,' Juliana \\ Fonseca Monteiro, ${ }^{2}$ Ana Carolina Hespanha, ${ }^{3}$ \\ José Fernando Ibañez, \\ 'Veterinary Surgeon of Orthopedics and Soft tissue Surgery, \\ Public Veterinary Hospital, Brazil \\ ${ }^{2}$ Veterinary Improvements in Small Animal Surgical Clinic, \\ Federal University of Paraná, Brazil \\ ${ }^{3}$ Veterinary Improvements in Small Animal Surgical Clinic, Public \\ Veterinary Hospital, Brazil \\ ${ }^{4}$ Small Animal Surgery Clinic, Federal University of Paraná, \\ Curitiba, Brazil
}

\begin{abstract}
Correspondence: Danilo Roberto Custódio Marques, Veterinary Surgeon of Orthopedics and Soft tissue Surgery, Public Veterinary Hospital, São Paulo, Brazil, Tel +55/4 3732 3925, Email danvetmarques@gmail.com
\end{abstract}

Received: October 22, 2017 | Published: November 22, 2017

\section{Introduction}

Tibial fractures are common in cats and complications are frequent. ${ }^{1-14}$ They occur more often in the middle and distal diaphysis, the latter accounting for $20 \%$ of all tibial fractures. ${ }^{3,4}$ The distal tibial diaphysis has some characteristics that influence bone healing following fracture. The craniomedial aspect of the tibia has limited muscle attachments, and the cortical: cancellous bone ratio is higher in the distal third than the proximal part of the bone meaning extra osseous blood supply to the distal tibial diaphysis is limited. ${ }^{1,2}$ It has been shown that local factors have an important contribution to play in non-union ${ }^{5}$ showed an incidence of non-union of $4.3 \%(18 / 422)$ in cats, with one of the highest incidences of non-union occurring in the tibia. The choice of fracture repair method depends on several factors and successful fracture healing requires proper planning of fixation. ${ }^{6-8}$ Creation of an optimal mechanical environment is very important for fracture healing, especially in fractures of the tibial diaphysis. ${ }^{9}$

Stimulation of bone cells to regenerate is largely influenced by the method of fixation. ${ }^{9}$ Small gaps between major fracture fragments are associated with higher strain and the use of plates and cerclage wire gives rigid stability, enhances cortical contact and leads to primary bone healing. ${ }^{10}$ Accurate restoration of anatomy allows early return to function, and results in shearing forces between the bone and the primary method of stabilization. With an absolutely stable fixation there is no change in shape or direction at the fracture interfaces, and secondary bone callus does not form. ${ }^{13}$ The rigidity of the repair with cerclage depends on how well the wires are tensioned and the accuracy of the fracture reconstruction i.e. the fit of the bones. ${ }^{7,8}$

The use of a locking compression plate minimizes impairment of the vascular supply to the bone because contact between bone and implant is limited. Internal fixation with a LCP can produce neutralization at the fracture site. ${ }^{11}$ Because of their fixed-angle stability, locking implants offer an increased resistance to screw pullout for both monocortical and biotical screws. ${ }^{17}$
This paper reports the use of a combination of cerclage wire and a neutralization plate in two cats, resulting in primary bone healing. Two non-pedigree male cats (cat 1 and 2), cat 1 weighing $4.1 \mathrm{~kg}$ and cat 2 weighing $3.9 \mathrm{~kg}$, were presented at the Veterinary Hospital of the Federal Univer- sity of Paraná following automobile trauma. General physical examination was unremarkable apart form orthopedic abnormalities. On orthopedic examination, instability, pain and crepitatus were found in the distal region of left tibia and fibula, indicative of fracture. Ventrodorsal and right lateral thoracic radiographs (to rule out pulmonary contusion) were made as well as craniocaudal and lateral views of the tibial and left fibula processes. One cat was presented with a closed tibial fracture. Pre-operative radiographs showed long oblique fractures of the distal third of the tibial diaphysis. The radiographs of the affected limbs of animals 1 and 2, are (Figure 1) (Figure 2), respectively.
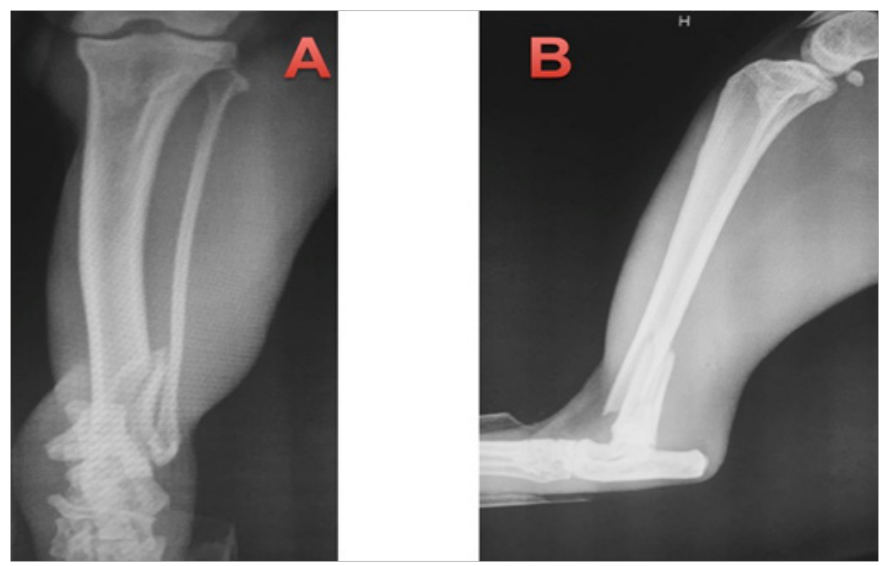

Figure I Cat I, with diagnosis of oblique fracture in the distal region of the left tibia and transverse fracture in the left distal fibula. (A) Craniocaudal projection showing oblique fracture in the distal tibia. (B) Mid-lateral projection with oblique fracture visible in the distal tibia. 


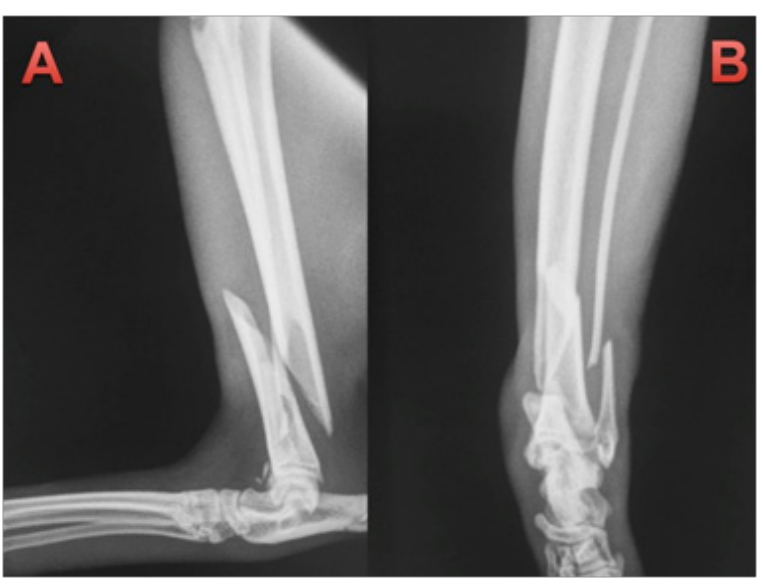

Figure 2 Cat 2, with diagnosis of oblique fracture in the distal region of the left tibia and transverse fracture in the left distal fibula. (A) Lateral-medial projection with oblique fracture in the left distal tibia. (B) Craniocaudal projection showing oblique fracture in the distal tibia.

Pre-operative examinations, complete blood counts and biochemistry profiles were performed, and analgesia was given at the time of admission. After blood samples had been collected for complete blood count and biochemistry, animals were premeditated with alfentanyl (60 micro $/ \mathrm{kg}$ IM) and acepromazine $(0.05 \mathrm{mg} / \mathrm{kg}$ IM), and anesthesia was induced with protocol (4-6mg/kg IV), and maintained with isoflurane in $100 \%$ oxygen. Analgesia was provided with target-controlled infusion of sufentanyl in combination with lidocaine and ketamine. Preoperatively methadone $(0.3 \mathrm{mg} / \mathrm{kg} \mathrm{IM})$ and meloxican $(0.1 \mathrm{mg} / \mathrm{kg} \mathrm{SC})$ was administered. Cephalothin $(30 \mathrm{mg} / \mathrm{kg}$, IV) was administered 15-30 minutes prior to initial skin incision and repeated every 90minutes during the procedure. The fracture in the distal third of the tibial diaphysis was accessed via a medial approach. After minimal blunt dissection, the fracture line was indirectly reduced and fixed with two looped cerclage wires. After fracture stabilization and compression with the cerclage wires a neutralization locking plate was applied over the primary construction. Plate size was selected according to the animal and bone size and at least 4 cortical screws were placed in the proximal and distal fracture fragments. On the medial malleolus a locked monocortical screw was placed to avoid articular penetration. Soft tissues were closed over the plate using a simple mattress pattern in 2/0 absorbable polydioxanone, followed by closure of the subcutaneous tissue using polydioxanone and skin closure with nylon.

Immediate post-operative radiographs were taken in cat 1 (Figure 3 ) and 2 (Figure 4), with follow-up radiographs at 21 days and 42days post-surgery. The cats were discharged the day after surgery, and reexamined for suture removal after 10days. Cephalexin $(30 \mathrm{mg} / \mathrm{kg}$ every 12 hours for 7 days orally), meloxicam $(0.05 \mathrm{mg} / \mathrm{kg}$ every 24 hours for 3 days orally), dipyrone $(25 \mathrm{mg} / \mathrm{kg}$ every 24 hours for 5 days, orally), tramadol hydrochloride $2 \mathrm{mg} / \mathrm{kg}$ every 12 hours for 5 days, orally) were prescribed post-operatively and a protective collar was dispensed to prevent the removal of the stitches. After 10days, the animals returned to HV-UFPR for removal of the stitches and orthopedic evaluation. Immediate post-operative radiographs showed excellent apposition and alignment of bone fragments. Plates were well positioned and fracture lines were not visible (Figure 2). The alignment, fragment fixation, apparatus placement and biological activity were good. The fracture line was not visible, thus showing compression of the fragments using cerclage. The plates have neutral function in both cases, therefore clerk as primary tutor and board as secondary tutor.

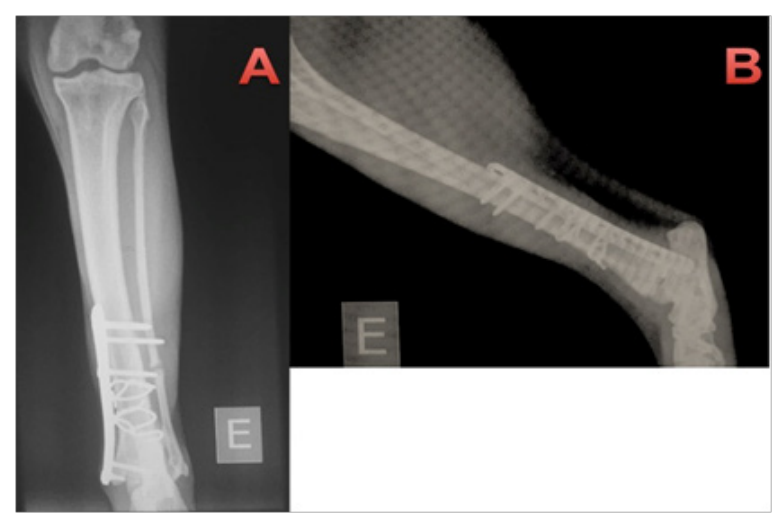

Figure 3 Immediate post-operative osteosynthesis of the left tibia of cat I. (A) Craniocaudal projection in which two cerclage wires were seen to cause compression of the bone fragments (fracture line not visible) and neutral blocked plate, using two screws locked in the distal fragment and three in proximal fragment. Fibular fracture, without implants. (B) Lateral-medial projection showing the same apparatuses as in $\mathrm{A}$, note fracture line not visible.
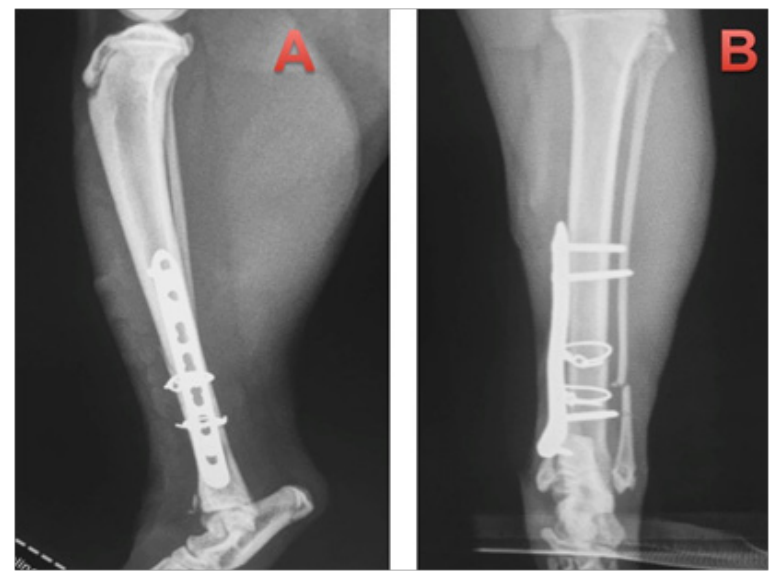

Figure 4 Immediate post-operative osteosynthesis of the left tibia of cat 2. (A) Mediolateral projection: projection showing two cerclages causing compression of the bone fragments (fracture line not visible) and neutral blocked plate, using two screws locked in distal fragment and three in proximal fragments. (B) Craniocaudal projection showing the same apparatuses as in letter $A$, again the line of the fracture is not visible, but fracture in the distal fibula can be seen.

Animals were weight bearing at the time of suture removal by 10days the postoperative. On the same day, the limb was examined for weight bearing, palpation at the surgery site, absence of crepitation and instability. At 21 days no callus was observed on control radiographs and the implants showed no signs of loosening in both cats. Further radiographs were taken at 42 and 63days, after which time patients were clinically discharged. One of the animals had a four month follow up in the postoperative period and secondary callus was not present in cat 1 or 2, in Figures 5 and 6, respectively. No clinical or orthopedic changes were found after 4months postoperatively (Figure 5) (Figure 6). Some previous studies have postulated that the high incidence of non-union in feline distal tibial fractures is related to a combination of unstable fixation, inadequate blood supply, excessive fracture gaps and interposition of soft tissue between fracture fragments. Compression of the fracture line provides continuous bone contact and thus minimizes strain, permitting primary healing. ${ }^{10-15}$ Restoration of the bone column by compression of the fracture line also results in rigid stability allowing the weight load to be shared between the bones and implant, ${ }^{10-13}$ and decreasing the chances of implant loosening. ${ }^{12}$ 

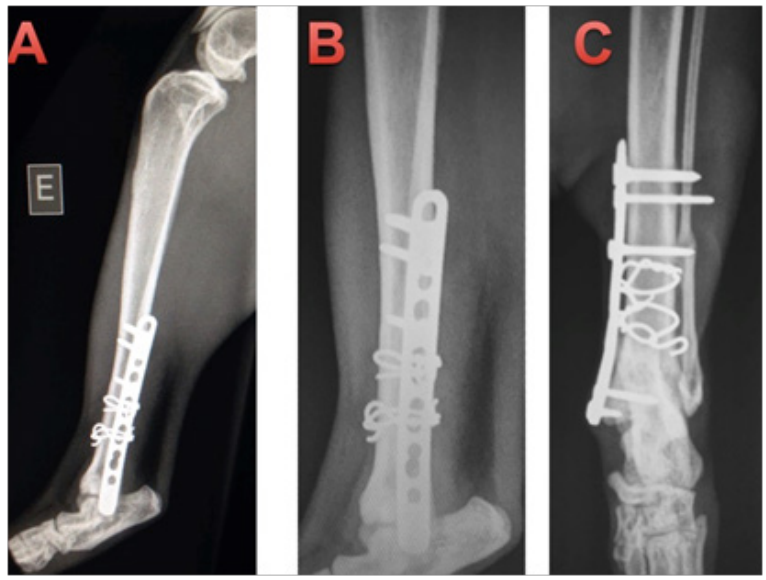

Figure 54 months postoperative of the cat $\mathrm{I}$. In all the images the bone consolidation in tibia has occurred without the production of primary bone callus, showing the total fragmentary compression caused by the cerclage. (A) Mid-lateral radiograph with cerclage wires and blocked plate with neutral function. (B) Close up showing primary bone callus. (C) Craiocaudal projection, showing cerclage.

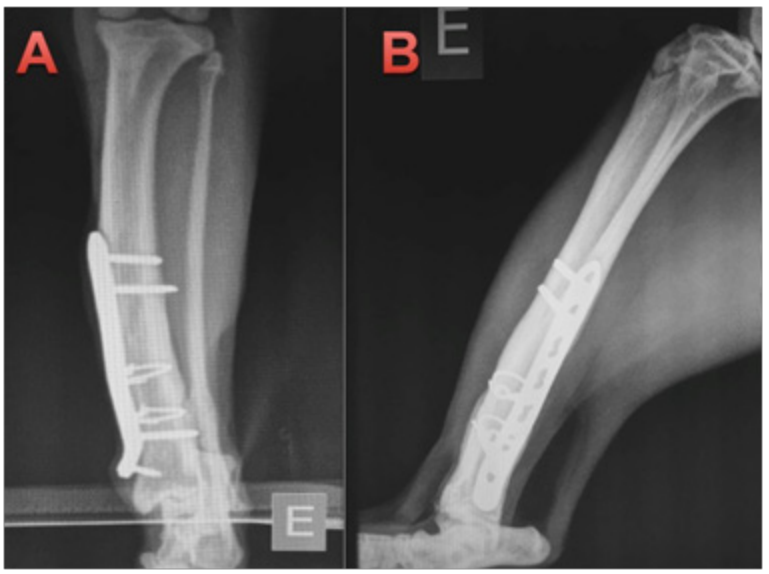

Figure 64 months postoperative of the cat I. In all the images the bone consolidation in tibia is seen without the production of primary bone callus, showing the total fragmentary compression caused by the cerclage. (A) Crabiocaudal projection showing bone cerclage (compression) and blocked plate with support function. Primary tibial callus is observed in tibia and secondary in tibia. (B) Medio-cranial projection with the same implants as A, showing primary bone callus.

A fast return to function is important to minimize time to clinical discharge, especially in none "in door" cats. ${ }^{1}$ Suggested that open internal fixation may be prudent for feline tibial diaphyseal fractures wherever practical. The reducible aspect of the long oblique noncomminuted fracture line as presented in this study permits open internal reduction with primary healing. Direct bone healing is histologically described by 'cutting cones' crossing the fracture gap, bypassing the intermediate stages of cartilaginous callus and endochondral ossification. ${ }^{16}$ The absence of callus formation on follow up radiographs in the cats in this study confirms primary bone healing. Cerclage wires maintain reduction of fracture fragments by tensional load compression. ${ }^{8}$ Fracture configuration is a very important consideration for successful use of cerclage wires. ${ }^{8}$ In the cases reported here the fracture plane did not coincide with the plane of the plate so the fracture could be secured with two cerclage wires instead of a lag screw and the plate functioned as a neutralization device. The locking plate systems are well known for producing angular stability allowing the use of fewer screws either side of the fracture. ${ }^{17}$

Distal fractures are very challenging because the fragments are often too small to allow four cortical screws to be place. ${ }^{17}$ Showed no significant difference in fixation failure between fractures stabilized with two, or more than two, biotical locking screws per main fragment. When used in combination with the cerclage wire, to provide primary stabilization, the locking plate added strength to the repair ${ }^{11}$ reported two femoral fractures treated with a combination of cerclage wires and a neutralizing LCP with successful alignment on post-operative radiographs. The combination of cerclage wires and a neutralizing plate can result in bone healing with the formation of primary bone callus in cats.

\section{Ethical approval}

All applicable international, national and/ or institutional guidelines for the care and use of animals were followed.

\section{Acknowledgements}

None.

\section{Conflict of interest}

The authors declare that they have no conflict of interest. The authors alone are responsible for the content and writing of paper.

\section{References}

1. Perry KL, Bruce M. Impact of fixation method on postoperative complication rates following surgical stabilization of diaphyseal tibial fractures in cats. Veterinary and Comparative Orthopaedics and Traumatology. 2015;28(2):109-115.

2. Glyde M, Arnett R. Tibial fractures in the dog and cat: options for management. Irish Veterinary Journal. 2006;59(5):290-295.

3. Voss K, Langley-Hobbs SJ, Montavon PM. Tibia and fibula. Feline Orthopedic Surgery and Musculoseskeletal Disease. 2009;39:491-505.

4. Seaman JA, Simpson AM. Tibial fractures. Clinical Techniques in Small Animal Practice. 2004;19(3):151-167.

5. Nolte DM, Fusco JV, Peterson ME. Incidence of and predisposing factors for nonunion of fractures involving the appendicular skeleton in cats: 18 cases (1998-2002). J Am Vet Med Assoc. 2005;226(1):76-85.

6. Chandler JC, Beale BS. Feline Orthopedics. Clinical Techniques in Small Animal Practice. 2002;17(4):190-203.

7. Van der Zee J. In vitro biomechanical comparison of the effects of the cerclage wires, as intramedullary pin and the combination thereof on an oblique osteotomy of the canine tibia. Veterinary and Comparative Orthopaedics and Traumatology. 2014;27(2):91-96.

8. Roe SC. Mechanical characteristics and comparisons of cerclage wires: Introduction of the double-wrap and loop/twist tying methods. Vet Surg. 1997;26(4):310-316.

9. Goodship AE, Kenwright J. The influence of induced micro movement upon the healing of experimental tibial fractures. J Bone Joint Surg Br. 1985;67(4):650-655.

10. Harari J. Treatments for feline long bone fractures. Veterinary Clinics: Small Animal Practice. 2002;32(4):927-947.

11. Haaland PJ, Sjöström L, Devor M, et al. Appendicular fracture repair in dogs using the locking compression plate system: 47 cases. Vet Comp Orthop Traumatol. 2009;22(4):309-315.

12. Hill FWG. A survey of bone fracture in the cat. Journal Small Animal Practice. 1977;18(7):457-463. 
13. Perren SM. Evolution of internal fixation of long bone fractures. The Bone \& Joint journal. 2002;86(8):1093-1110.

14. Dugat D, Rocha M, Ritchey J. Quantitative analysis of the intramedullary arterial supply of the feline tibia. Veterinary and Comparative Orthopaedics and Traumatology. 2011;24(5):313-319.

15. Maxwell M, Horstman CL, Crawford RL, et al. The effects of screw placement on plate strain in $3.5 \mathrm{~mm}$ dynamic compression plate and limited-contact dynamic compression plate. Veterinary and Comparative Orthopaedics and Traumatology. 2009;22(2):125-131.
16. Barry S. Non-steroidal anti-inflammatory drug inhibit bone healing: A review. Veterinary and Comparative Orthopaedics and Traumatology. 2010;23(6):385-392.

17. Vallefuoco R, Pommellet HLe, Savin A, et al. Complications of appendicular fracture repair in cats and small dogs using locking compression plates. Veterinary and Comparative Orthopaedics and Traumatology. 2016;29(1):46-52. 